\title{
Developing the Community reporting system for FOODBORNE OUTBREAKS
}

\author{
A Gervelmeyer (andrea.gervelmeyer@bfr.bund.de) ${ }^{1}$, M Hempen², U Nebel ${ }^{1}$, C Weber ${ }^{1}$, S Bronzwaer ${ }^{2}$, A Ammon ${ }^{3}$, Pia Makela $^{2}$ \\ 1. Bundesinstitut für Risikobewertung (Federal Institute for Risk Assessment), Berlin, Germany \\ 2. European Food Safety Authority, Parma, Italy \\ 3. European Centre for Disease Prevention and Control, Stockholm, Sweden
}

Investigating and reporting of foodborne outbreaks became mandatory with Directive 2003/99/EC. In 2006 and 2007 the Community reporting system for foodborne outbreaks was further developed in an interdisciplinary approach, which is described in this paper. This involved experts on investigating and reporting foodborne outbreaks as well as experts on communicable diseases in addition to the European Food Safety Authority (EFSA) Task Force for Zoonoses Data Collection, the European Centre for Disease Prevention and Control (ECDC) Advisory Forum and representatives of ECDC, the World Health Organization (WHO), the World Organization for Animal Health (OIE) and the European Commission. European Union Member States participated in a survey regarding their national reporting systems and the needs for information on foodborne outbreaks at the Community level. The acceptability, the functionality and the data quality of the current reporting system were evaluated. The results were used to propose new variables on which data should be reported. Pick-lists were developed to facilitate reporting and better integration of the Community system with Member States' reporting systems. The new system is expected to yield better quality data on foodborne outbreaks relevant for risk assessment and risk management while reducing the work load for Member States.

Introduction

Protection of human health against diseases and infections transmissible directly or indirectly between animals and humans (zoonoses) is of paramount importance. In order to assess the priorities for preventive action against zoonoses in the European Community, the European Union (EU) Member States have been obliged since the end of 1993 to collect data on the trends and sources of zoonotic infections in the human population and on the occurrence of zoonotic agents in animals, food, and animal feed [1].

Foodborne outbreaks, if thoroughly investigated, provide the possibility to identify the pathogen, the food vehicle involved and the factors in the preparation and handling of food that contributed to the outbreak. Therefore, it was considered appropriate to make provision for such investigations and for close cooperation between the various authorities when a new "Zoonoses directive" was developed in 2003. The Directive 2003/99/EC of the European Parliament and of the Council on monitoring of zoonoses and zoonotic agents [2] requests the EU Member States to investigate foodborne outbreaks and to transmit each year to the Commission a summary report of the results of the investigations carried out. The European Food Safety Authority (EFSA), who is assigned the task to collate, analyse and report the data collected, developed a reporting system for foodborne outbreaks in 2003. When the reporting of foodborne outbreaks became mandatory in 2005, EFSA with the assistance of its Foodborne Outbreak Contractor, the Bundesinstitut für Risikobewertung (Federal Institute for Risk Assessment, BfR), and in collaboration with the European Centre for Disease Prevention and Control (ECDC) further developed the Community reporting system for foodborne outbreaks.

This report describes the activities undertaken in this context and summarises their results.

\section{Methods}

A survey was conducted with the aim to investigate the national reporting systems for foodborne outbreaks currently in place in the Member States and to establish the need for collecting further information on foodborne outbreaks at the Community level.

Following this, the current Community reporting system for foodborne outbreaks was evaluated regarding its acceptability, data quality and sensitivity.

The results of the questionnaire survey and the evaluation were used in further developing the Community reporting system for foodborne outbreaks.

\section{Questionnaire survey}

Two questionnaires were prepared for the survey. In the first questionnaire, the recipients were asked to describe the structure of their national reporting system for foodborne outbreaks, how foodborne outbreaks were investigated and results of those investigations reported. In the second questionnaire, the recipients were asked to prioritise proposed objectives of the improved Community reporting system for foodborne outbreaks and to list other objectives they considered important. They were also requested to prioritise possible new parameters on which data should be reported to the Community level through the improved Community reporting system.

Both questionnaires were sent to representatives of the EU Member States and other European countries participating in the EU data collection (30 countries in total were contacted). They were asked to further distribute the questionnaires among the relevant institutes and persons in charge of the reporting of foodborne outbreaks in their country, and to return the completed forms. In 
addition, representatives of the European Commission, a number of international organisations and networks, and EFSA scientific panels were asked to complete the second questionnaire only.

Completed questionnaires were analysed at the BfR. The absolute and relative frequencies were calculated for all response options given in the questionnaires. In addition, for each of the general objectives and parameters of the improved reporting system given in the second questionnaire on information needs, scores were calculated by multiplying the frequency with which an objective had been assigned a priority level with the rank of the priority level ("High priority" = 2, "Low priority" = 1, "No need" =0).

\section{Evaluation of the reporting system}

The current reporting system was evaluated by the Foodborne Outbreak Contractor (BfR) by assessing the data on foodborne outbreaks occurring in 2005 and submitted by Member States to the system before August 2006. The acceptability of the system was evaluated by calculating the overall participation rate of Member States, the submission rate for the respective reporting forms of the system and the completeness (represented by nonblank data fields) for all fields of the reporting forms. Data quality was evaluated by assessing the validity and the completeness of data submitted through the reporting forms. The sensitivity of the system relative to the sensitivity of national reporting systems was estimated for a subset of countries by comparing data on foodborne outbreaks submitted to the Community reporting system with data on foodborne outbreaks published in national bulletins, national annual reports and peer-reviewed journals.

\section{Developing the reporting system further}

A working group on foodborne outbreaks was set up by EFSA with experts on food safety and public health as well as representatives of ECDC, the World Health Organization (WHO), the World Organization for Animal Health (OIE) and the Directorate General for Health and Consumer Protection of the European Commission. Its task was to identify the need for reporting more information on foodborne outbreaks at the Community level and the availability of this data in the national reporting systems in Member States. The working group also analysed the results and the functionality of the current Community reporting system for foodborne outbreaks.

Based on their work, as well as the results of the questionnaire survey and the evaluation of the reporting system, a proposal for the improved reporting system was drafted. Subsequently, both the Task Force on Zoonoses Data Collection of EFSA and the Advisory Forum of ECDC were consulted and provided their comments, and the draft document was accordingly adjusted.

\section{Results}

\section{Questionnaire survey}

Twenty-six countries (response rate $87 \%$ ) provided information on current national reporting system of foodborne outbreaks through the first questionnaire (27 systems were described, as one country provided information on two systems). In addition, 32 pick-lists of possible entries for a range of variables used in the national reporting systems were provided by 13 countries [3].

Thirty-five completed copies of the second questionnaire on information needs were received from representatives of 26 countries and two international bodies.

\section{Foodborne outbreak reporting systems in place in the countries}

All respondents confirmed that their country operated a reporting system for foodborne outbreaks, including waterborne outbreaks.
All countries covered outbreaks caused by bacteria, viruses and parasites $(n=26)$. Information on outbreaks caused by toxins were collected by 22 systems and data on outbreaks caused by chemicals by 10 systems. The majority of the national reporting systems were complex and involved several authorities. Eight countries claimed that there was close co-operation between public health and food safety/ veterinary authorities while five countries reported the establishment of national commissions or platforms for foodborne outbreaks aiming at improving the exchange of information and collaboration between the public health, veterinary and food safety authorities on zoonoses and, specifically, on foodborne outbreaks. Most countries recorded information on the number of human cases, the number of hospitalisations and deaths related to the outbreak. Many of them also differentiated between laboratoryconfirmed and epidemiologically linked human cases and included age and gender of the cases (Table 1 ).

The incriminated food item could be reported as a free text in 17 of the systems. Five systems provided a default list with food items or categories from which the appropriate item could be picked and five systems offered both options. Most systems recorded the place of consumption and the place of preparation of the incriminated food, while the methods of food processing and food preparation were registered less frequently (Table 2). The most frequently stated shortcomings of the national reporting systems were the varying depths of outbreak investigations and the difficulties in tracing back the incriminated food.

\section{Information needs at the Community level}

The three objectives for data collection that received the highest overall score from all respondents were the identification and the monitoring of the food vehicles, the causative agents and the risk factors of foodborne outbreaks. Altogether 29 variables on which data should be collected through the improved Community foodborne outbreak reporting system were offered for prioritisation.

\section{T A B L E E}

Information on human cases involved in foodborne outbreaks covered by the national reporting systems $(n=27)$

\begin{tabular}{|l|c|c|}
\hline \multirow{2}{*}{ Variable } & \multicolumn{2}{|c|}{ Systems } \\
\cline { 2 - 3 } & $n$ & $\%$ \\
\hline Number of human cases in the outbreak & 27 & 100 \\
\hline Number of deaths caused by the outbreak & 25 & 93 \\
\hline Number of cases hospitalised & 24 & 89 \\
\hline $\begin{array}{l}\text { Number of laboratory confirmed human cases in } \\
\text { the outbreak }\end{array}$ & 22 & 82 \\
\hline $\begin{array}{l}\text { Number of epidemiologically confirmed cases in } \\
\text { the outbreak }\end{array}$ & 18 & 67 \\
\hline Age of the person affected & 18 & 67 \\
\hline Gender of the persons affected & 18 & 67 \\
\hline Number of persons at risk & 17 & 63 \\
\hline $\begin{array}{l}\text { Number of laboratory confirmed clinical* cases } \\
\text { in the outbreak }\end{array}$ & 14 & 52 \\
\hline $\begin{array}{l}\text { Number of laboratory confirmed asymptomatic } \\
\text { cases in the outbreak }\end{array}$ & 7 & 26 \\
\hline $\begin{array}{l}\text { Number of person-days-in-hospital caused by the } \\
\text { outbreak }\end{array}$ & 3 & 11 \\
\hline
\end{tabular}

$\mathrm{n}=$ number of national reporting systems collecting data on the variable $\%=$ percentage of all reporting systems

= symptomatic 
Among the variables related to human cases, the following were considered to be most important: the number of human cases and deaths, the beginning and the end date as well as the location of the outbreak and the type of the outbreak. Of the variables related to the food vehicle, the identification of the food vehicle, its origin, the evidence for incriminating the food vehicle, the places of food preparation and consumption, the origin of the contamination of the food vehicle, the factors contributing to its contamination as well as the results of the laboratory analysis of the food vehicle were considered to be the most relevant variables (Table 3).

\section{Evaluation of the reporting system}

The web-based reporting system for foodborne outbreaks developed by EFSA in 2003 and used until 2007 provided a table form to capture information on the total number of outbreaks per year, the number of human cases and deaths in these outbreaks,

T A B L E

Information on factors regarding the incriminated food item collected by the national reporting systems $(n=27)$

\begin{tabular}{|l|c|c|}
\hline \multirow{2}{*}{ Variable } & \multicolumn{2}{|c|}{ Systems } \\
\cline { 2 - 3 } & $n$ & $\%$ \\
\hline Place of consumption & 26 & 96 \\
\hline Place of food preparation & 23 & 85 \\
\hline Factors contributing to contamination of the food & 23 & 85 \\
\hline $\begin{array}{l}\text { Factors contributing to survival/multiplication of } \\
\text { the agent in the food }\end{array}$ & 21 & 78 \\
\hline Origin of contamination of the food & 20 & 74 \\
\hline $\begin{array}{l}\text { Origin of incriminated food (i.e. imported or } \\
\text { national product) }\end{array}$ & 18 & 67 \\
\hline Method of food preparation & 15 & 56 \\
\hline Method of food processing & 14 & 52 \\
\hline $\begin{array}{l}\text { Reasons not allowing identification of origin of } \\
\text { food contamination }\end{array}$ & 12 & 44 \\
\hline
\end{tabular}

$\mathrm{n}=$ number of national reporting systems collecting data on the variable $\%=$ percentage of all reporting systems the causative agents of the outbreaks, the foodstuffs implicated as vehicles of the causative agents, the location of exposure of the human cases to the contaminated food vehicle and the contributory factors, i.e. the factors contributing to the contamination of the incriminated food. In addition, a text form was provided by the web-based reporting system to capture information on the national system in place for identification, epidemiological investigations and reporting of foodborne outbreaks, the types of outbreaks covered by the system, the national evaluation of the reported outbreaks with respect to relevance of the different causative agents, food categories and the agent/food category combinations and an evaluation of the severity and clinical picture of the human cases, the description of single outbreaks of special interest and on the control measures or other actions taken to improve the situation. All data fields except those for the information on the 'causative agent', which could be chosen from a pick-list with variable degrees of detail (speciation and subtyping information), were free text fields.

By August 2006, of the 26 countries eligible for reporting (25 EU Member States plus Norway), 24 countries (23 EU MS and Norway) submitted data on foodborne outbreaks which had occurred in 2005, resulting in $92 \%$ participation rate. The table form was used by 21 EU MS and Norway ( $n=22,85 \%$ ), whereas the text form was submitted by 19 EU MS and Norway $(n=20$, $77 \%)$. In all, 972 table-form reports were submitted, the majority of which contained information on individual outbreaks $(n=826$, $85 \%$ ), whereas in less than one-fifth of the reports ( $n=146,15 \%$ ) information on more than one outbreak was aggregated.

Information on the causative agent at the genus-level was provided in all aggregated and all individual reports. All reports also contained information about the type of outbreak ("general outbreak" or "family outbreak"). The number of human cases was given in $99 \%$ of individual and $96 \%$ of aggregated outbreak reports. Data on the vehicle of the outbreak, that is the foodstuff incriminated for causing the outbreak, was available in $92 \%$ of the individual outbreak reports but only in $78 \%$ of the aggregated outbreak reports. Information on the "location of exposure" was

T A B L E 3

Prioritisation of objectives for the Community foodborne outbreak reporting system by the respondents (n=35)

\begin{tabular}{|c|c|c|c|c|c|c|c|c|c|}
\hline \multirow{2}{*}{ Objective } & \multicolumn{2}{|c|}{ High priority } & \multicolumn{2}{|c|}{ Low priority } & \multicolumn{2}{|c|}{ No need } & \multicolumn{2}{|c|}{ Other } & \multirow{2}{*}{ Score } \\
\hline & n & $\%$ & n & $\%$ & n & $\%$ & n & $\%$ & \\
\hline Gather information on and monitor the vehicles of food-borne outbreaks & 31 & 88 & 3 & 9 & 1 & 3 & 0 & 0 & 65 \\
\hline Gather information on and monitor the agents causing food-borne outbreaks & 31 & 88 & 3 & 9 & 1 & 3 & 0 & 0 & 65 \\
\hline Gather information on and monitor risk factors ${ }^{\star}$ for food-borne outbreaks & 30 & 85 & 4 & 12 & 1 & 3 & 0 & 0 & 64 \\
\hline Monitor trends in agents causing food-borne outbreaks & 28 & 79 & 6 & 18 & 1 & 3 & 0 & 0 & 62 \\
\hline Identify new agents causing food-borne outbreaks & 29 & 82 & 3 & 9 & 2 & 6 & 1 & 3 & 61 \\
\hline Provide comparable data on food-borne outbreaks & 26 & 74 & 8 & 23 & 1 & 3 & 0 & 0 & 60 \\
\hline Evaluate the impact of control measures taken & 25 & 71 & 9 & 26 & 1 & 3 & 0 & 0 & 59 \\
\hline Identify new vehicles of food-borne outbreaks & 24 & 69 & 9 & 26 & 2 & 6 & 0 & 0 & 57 \\
\hline Monitor trends in vehicles involved in food-borne outbreaks & 22 & 62 & 12 & 35 & 1 & 3 & 0 & 0 & 56 \\
\hline $\begin{array}{l}\text { Gather information on and monitor special risk groups of consumers for food- } \\
\text { borne outbreaks }\end{array}$ & 19 & 56 & 13 & 35 & 3 & 9 & 0 & 0 & 51 \\
\hline
\end{tabular}

$\mathrm{n}=$ number of respondents assigning the objective to the priority level; \%= percentage of all respondents; score= number of respondents assigning the objective to a given priority level multiplied with the rank of the priority level ("High priority" = 2, “Low priority" = 1 , "No need" = 0); * risk factors = host factors and factors contributing to the contamination of the incriminated food 
given in $95 \%$ of the individual and $75 \%$ of the aggregated outbreak reports respectively (Table 4 ).

Most countries provided some information on their reporting systems, on the evaluation of the national situation regarding foodborne outbreaks as well as a description of the types of outbreaks covered by their reporting systems (between 80 to $90 \%$ completeness) through the text form.

The quality of the submitted data was assessed separately for data submitted through the table form and for data submitted through the text form. In the individual outbreak reports submitted through the table form, most of the data provided on the type of evidence and the location of exposure were submitted under the corresponding field of the table (96 and $90 \%$ of the relevant entries). In contrast, only $70 \%$ of the information on the food vehicle of the outbreak was submitted under the corresponding field ("Source"), and only slightly more than half of the information on contributing factors was reported under the field "Contributing factors" (Table 5).
For all 146 aggregated outbreak records submitted in the table form, whenever information on the incriminated food vehicle was given it was entered in the corresponding field of the table. The same applies to the information submitted on the location of exposure. In contrast, only $76 \%$ of the information on contributing factors was provided under the corresponding field.

A large proportion of the 20 completed text forms contained the requested information on the authorities and institutions involved in investigating and reporting foodborne outbreaks, on their roles and responsibilities, and on mandatory and voluntary activities in this field ( 75 to $80 \%$ ). Approximately half of the text forms contained the requested information on the relevance of the agents involved in the reported foodborne outbreaks (60\%) and the types of outbreaks covered by the system (50\%). Information on the trends observed in the number of outbreaks and cases, the relevance of the places of food production and preparation as well as the evaluation of the severity and clinical pictures of the human cases was provided less frequently (range 5-35\% completeness).

T A B L E 4

Completeness of outbreak records submitted in the table forms $(\mathbf{n = 9 7 2})$

\begin{tabular}{|c|c|c|c|c|c|c|}
\hline \multirow[b]{2}{*}{ Data field } & \multicolumn{2}{|c|}{ All outbreak records ( $n=972$ ) } & \multicolumn{2}{|c|}{ Aggregated outbreak records ( $n=146$ ) } & \multicolumn{2}{|c|}{ Individual outbreak records ( $n=826$ ) } \\
\hline & $\begin{array}{l}\text { No. non-blank } \\
\text { fields }\end{array}$ & Completeness (\%) & $\begin{array}{l}\text { No. non-blank } \\
\text { fields }\end{array}$ & Completeness (\%) & $\begin{array}{l}\text { No. non-blank } \\
\text { fields }\end{array}$ & Completeness (\%) \\
\hline Causative agent & 972 & 100 & 146 & 100 & 826 & 100 \\
\hline Causative agent species & 797 & 82 & 120 & 82 & 677 & 82 \\
\hline Causative agent Subtype & 304 & 31 & 49 & 34 & 255 & 31 \\
\hline Outbreak type & 971 & 100 & 146 & 100 & 824 & 100 \\
\hline Number of persons ill & 959 & 99 & 140 & 96 & 819 & 99 \\
\hline Number of persons who died & 653 & 67 & 112 & 77 & 541 & 65 \\
\hline Number of persons in hospital & 732 & 75 & 112 & 77 & 620 & 75 \\
\hline Source $^{\star}$ & 878 & 90 & 114 & 78 & 764 & 92 \\
\hline Level of confirmation of source* & 784 & 81 & 79 & 54 & 689 & 83 \\
\hline Type of evidence & 576 & 59 & 63 & 43 & 513 & 62 \\
\hline Location of exposure & 897 & 92 & 110 & 75 & 787 & 95 \\
\hline Contributing factors & 382 & 39 & 21 & 14 & 361 & 44 \\
\hline Comment & 80 & 8 & 24 & 16 & 56 & 7 \\
\hline Footnote & 212 & 22 & 16 & 11 & 196 & 24 \\
\hline
\end{tabular}

*source = implicated food

T A B L E 5

Distribution of information of individual outbreak records $(n=826)$ in corresponding and non-corresponding fields of the table form

\begin{tabular}{|c|c|c|c|c|c|}
\hline \multirow{2}{*}{ Thematic area } & \multicolumn{2}{|c|}{$\begin{array}{l}\text { Requested information provided in } \\
\text { corresponding field }\end{array}$} & \multicolumn{2}{|c|}{$\begin{array}{l}\text { Requested information provided in } \\
\text { other field }\end{array}$} & \multirow{2}{*}{$\begin{array}{c}\text { Requested information } \\
\text { provided total } \\
\text { n } \\
\end{array}$} \\
\hline & $n$ & $\%$ & $n$ & $\%$ & \\
\hline Source* & 712 & 70 & 303 & 30 & 1015 \\
\hline Location of exposure & 713 & 90 & 80 & 10 & 793 \\
\hline Type of evidence & 279 & 96 & 11 & 4 & 290 \\
\hline Contributing factors & 146 & 55 & 119 & 45 & 265 \\
\hline
\end{tabular}

*source = implicated food 


\section{Sensitivity analysis}

The sensitivity of the reporting system was assessed for a subset of countries (Denmark, France, Germany, Ireland, Norway, Sweden, United Kingdom) by comparing individual records of outbreaks occurring in 2005 and reported to EFSA (EFSA dataset, $n=229$ ) with reports on individual foodborne outbreaks occurring in 2005 and published in national bulletins, annual reports or peer-reviewed journals (national dataset, $n=124$ ). Information on the causative agent and the type of outbreak was complete in both data sets. There was little difference between the levels of completeness for the number of human cases $197 \%$ in the EFSA and $96 \%$ in the national data set), the place of exposure $(85 \%$ and $84 \%$ respectively), the incriminated food (54\% and $63 \%$ respectively), the type of evidence ( $40 \%$ and $37 \%$ respectively) and the food processing information ( $26 \%$ and $28 \%$ respectively). Information on the number of deaths and the number of hospitalisations was more complete in the EFSA data set with $43 \%$ and $34 \%$ respectively as compared to the national data set with a completeness of $8 \%$ each. The national data set was more complete than the EFSA data set with regards to subtyping information (85\% as compared to $65 \%$ ), species information for food of animal origin (23\% as compared to $18 \%$ ), and contributing factors ( $15 \%$ as compared to $8 \%$ ).

Thirty-nine identical outbreaks were identified in the EFSA and the national dataset through matching of the information on the parameters "reporting country", "causative agent", "number of cases" and "food vehicle". For most of these outbreaks the level of detail of the information provided on the species for food of animal origin, on the place of exposure and on processing of incriminated food was the same in the EFSA and the national dataset (92\%, 87\% and $82 \%$ respectively). $50 \%$ of the reports contained information on the type of evidence only in the EFSA data set, whereas for $37 \%$ of the outbreak records information on contributing factors was reported exclusively in the national data set [4].

\section{Developing the reporting system further}

Taking into consideration the results of the survey and the evaluation of the current system, a proposal for a new foodborne outbreak reporting system was drafted. This proposal was subsequently accepted by the participating countries through the EFSA Task Force on Zoooses Data Collection and the ECDC Advisory Forum. The system has been used in May 2008 to report data from 2007.

Its main objectives are to assess the trends in the number and size of foodborne outbreaks and the share of outbreaks related to different causative agents [5]. It should also collect information on the severity of disease in the human cases involved; the importance of different food categories as vehicles of foodborne outbreaks and the risk factors contributing to the occurrence of foodborne outbreaks. The scope of the new system has been set to cover foodborne outbreaks caused not only by zoonotic agents, but by any virus, bacterium, algae, fungus, parasite, and their products, such as toxins and biological amines (e.g. histamine) as well as foodborne outbreaks where the causative agent remains unknown. Foodborne outbreaks caused by chemical agents are, however, not covered at this stage. Outbreaks caused by ingestion of drinking water are also considered foodborne since drinking water is defined as food in Regulation 178/2002/EC. An additional table form capturing the number of foodborne outbreaks, distinguishing between possible and verified foodborne outbreaks, has been introduced. Possible foodborne outbreaks are outbreaks compatible with descriptive epidemiological evidence alone including also outbreaks where the causative agent is unknown. Their number should be reported by causative agent, including the option "unknown agent", in the new table. The original table form should only be used to report details on verified outbreaks, i.e. outbreaks compatible with descriptive epidemiological evidence and laboratory detection of the causative agent in implicated food or analytical epidemiological evidence or both. The table has been modified by adding pick-lists for most of the variables. In addition to selecting the implicated foodstuff category from a pick-list, a free text field can be used to define the foodstuff in more detail, e.g. to submit details on the animal or plant species the food was made from and the treatment of the food. Two new variables have been added to the table to collect information on the place where the contamination or the mishandling of the implicated food occurred ("place of origin of problem") and on the origin of foodstuff, e.g. whether the implicated foodstuff originated from the domestic market, from intra-community trade or was imported from outside the EU. A comprehensive manual containing definitions of all terms included in the pick-lists as well as examples has been prepared to facilitate reporting. In April 2008 EFSA, in collaboration with ECDC, organised a training course in the new system for relevant officers of the countries participating in reporting.

\section{Discussion and conclusion}

The responses received through the questionnaire survey show that the vast majority of the national foodborne outbreak reporting systems in the EU provide the information that is requested pursuant Article 9 (1) of the Zoonoses Directive (Annex IV, E) [2]. In fact, many of the national systems collect complementary information on a number of variables. It is particularly encouraging to note that already many national systems collect detailed data on the incriminated food vehicles, on the causative agents, on the human cases and on the contributing factors. This could contribute to reaching the objectives of the Community reporting system considered most important by the survey respondents, i.e. the identification and the monitoring of the vehicles, the causative agent and the risk factors involved in foodborne outbreaks. However, when interpreting the results of the questionnaire on information needs it should be taken into account that the responses might have been influenced at least partially by the countries' capacities to collect the respective data. For example, the fact that collection of data on the method of food processing or on the origin of the food contamination ranked relatively low on the priority list is probably related to difficulties in tracing back the origin of foodstuffs and establishing this kind of information.

The evaluation of the Community reporting system revealed that its acceptability in general was very high as reflected by the high rates of participation and submission as well as the high proportion of completeness of most data fields. Also the sensitivity assessment indicated that the Community systems captured almost all foodborne outbreaks reported in national reports or peerreviewed journals and it collected sufficient detail of information available on most variables. With regard to subtyping information, which was less frequently captured by the EFSA system, it might be useful to consider whether reporting this type of data could be further simplified in the EFSA system. However, the results of the sensitivity assessment should be interpreted with some caution as the countries included in this evaluation have well established foodborne outbreak reporting systems and might not be representative for all EU Member States. 
The fact that a considerable fraction of the requested information is not reported in the corresponding data field of the current system makes the analysis of the reported data difficult. This is further aggravated by the occurrence of spelling variations (e.g. "restaurant" versus "restarant") and synonyms (e.g. "kindergarten" vs. "day care center") inherent in text data reporting. While spelling variations and the use of synonyms can be obviated by introducing list fields instead of free-text fields, the frequent misplacement of information in another than the intended field also indicates that clearer instructions and further explanations might be needed on the kind of information requested in each field of the reporting form.

The Community foodborne outbreak reporting system was developed further taking into account the existing structures, variables and pick-lists of Member States' national systems as well as other reporting systems, such as the WHO surveillance system for control of foodborne infections and intoxications in Europe [6].

This should not only harmonise, but also make the reporting of foodborne outbreaks easier for Member States. Another move into this direction is the introduction of the possibility to upload national data in bulk using XML-format. Through the differentiation between possible and verified foodborne outbreaks in the new system the quantity of data to be reported should be less, as detailed information is only requested for verified outbreaks. The data on verified outbreaks will be used to characterise the nature of foodborne outbreaks in the Community and to carry out in depthanalysis of the involved food vehicle-causative agent combinations. At the same time, the system should allow to study the overall extent and impact of foodborne outbreaks in the Community by additionally capturing the number of possible outbreaks. Detailed definitions for all variables have been established. They have been agreed upon by experts from both veterinary and public health. The introduction of pick-lists for most variables will facilitate both the manual inputting of data as well as the uploading of data in bulk. Together with the introduction of definitions, this will lead to a harmonisation of reporting and ease the analysis of the reported data. Possible problems with misunderstanding the meaning of the values in the pick-list should be minimal because of the provision of comprehensive explanations and examples in the reporting manual and extensive online-user-guidance provided by the web-based system.

Because of its higher level of integration with other existing reporting systems, its increased simplicity and, therefore, higher acceptability the new Community foodborne outbreak reporting system is expected to yield better quality data on foodborne outbreaks. This will hopefully increase the availability of relevant data for food safety risk assessment critical for identifying priorities for control and monitoring programmes.

\section{Acknowledgements}

Members of the Working Group on foodborne outbreaks included Katharina Alpers, Robert Koch-Institut, Germany, Andrea Ammon, ECDC, Stef Bronzwaer, EFSA, Daniel Chaisemartin, OIE, Kris De Smet, DG SANCO, Katelijne Dierick, Scientific Institute of Public Health, Belgium, Andrea Gervelmeyer, Bundesinstitut für Risikobewertung, Germany, Michaela Hempen, Bundesinstitut für Risikobewertung, Germany, Gloria Hernandez Pezzi, Instituto de Salud Carlos III, Spain, Marika Hjertqvist, Swedish Institute for Infectious Disease Control, Sweden, Judith Hilton, Food Standards Agency, United Kingdom, Franz Karcher, DG SANCO, Hilde Kruse, WHO, Danilo Lo Fo Wong, National Food Institute, Denmark, Pia Mäkelä, EFSA, Antonio Petrini, OIE, Tobin Robinson, EFSA.

\section{References}

1. Council Directive 92/117/EEC of 17 December 1992 concerning measures for protection against specified zoonoses and specified zoonotic agents in animals and products of animal origin in order to prevent outbreaks of foodborne infections and intoxications, Official Journal of the European Communities 1993; L062: 15/03/1993, pp. 38-48. Available from: http://eur-lex. europa.eu/LexUriServ/LexUriServ.do?uri=CELEX:31992L0117:EN:HTML

2. Directive 2003/99/EC of the European Parliament and of the Council of 17 November 2003 on the monitoring of zoonoses and zoonotic agents, amending Council Decision 90/424/EEC and repealing Council Directive 92/117/EEC, Official Journal L 325 , 12/12/2003 P. 0031 - 0040. Available from: http://eurlex.europa.eu/LexUriServ/LexUriServ.do?uri=CELEX:32003L0099:EN:HTML

3. Report on foodborne outbreak reporting systems in place in the Member States of the European Union and on needs for information on foodborne outbreaks in the European Community - results of a questionnaire survey. The EFSA Journal. 2007;577:1-37.

4. Report on evaluation of the Community reporting system for foodborne outbreaks under Directive 2003/99/EC. The EFSA Journal. 2007;131:1-40.

5. Report from the Task Force on Zoonoses Data collection on harmonising the reporting of foodborne outbreaks through the Community reporting system in accordance with Directive 2003/99/EC. The EFSA Journal. 2007;123:1-16.

6. Schmidt K, Gervelmeyer A. Editors. 8th Report of the WHO Surveillance Programme for Control of Foodborne Infections and Intoxications in Europe, 1999-2000.

This article was published on 6 November 2008

Citation style for this article: Gervelmeyer A, Hempen M, Nebel U, Weber C, Bronzwaer S, Ammon A, Makela P. Developing the Community reporting system for foodborne outbreaks. Euro Surveill. 2008;13(45):pii=19029. Available online: http://www.eurosurveillance.org/ ViewArticle.aspx?ArticleId $=19029$ 